\title{
Quantum dimer models and effective Hamiltonians on the pyrochlore lattice
}

\author{
R. Moessner, ${ }^{1}$ S. L. Sondhi ${ }^{2}$ and M. O. Goerbig ${ }^{3}$ \\ ${ }^{1}$ Laboratoire de Physique Théorique de l'Ecole Normale Supérieure, CNRS-UMR8549, Paris \\ ${ }^{2}$ Department of Physics, Princeton University, Princeton, New Jersey and \\ ${ }^{3}$ Laboratoire de Physique Théorique et Hautes Energies, \\ CNRS-UMR 7589, Université Paris 6 et 7, Paris
}

(Dated: March 22, 2018)

\begin{abstract}
We study a large- $N$ deformation of the $S=1 / 2$ pyrochlore Heisenberg antiferromagnet which leads to a soluble quantum dimer model at leading non-trivial order. In this limit, the ground state manifold - while extensively degenerate - breaks the inversion symmetry of the lattice, which implies a finite temperature Ising transition without translational symmetry breaking. At lower temperatures and further in the $1 / N$ expansion, we discuss an effective Hamiltonian within the degenerate manifold, which has a transparent physical interpretation as representing dimer potential energies. We find mean-field ground states of the effective Hamiltonian which exhibit translational symmetry breaking. The entire scenario offers a new perspective on previous treatments of the $\mathrm{SU}(2)$ problem not controlled by a small parameter, in particular showing that a mean-field state considered previously encodes the physics of a maximally flippable dimer configuration. We also comment on the difficulties of extending our results to the $\mathrm{SU}(2)$ case, and note implications for classical dimer models.
\end{abstract}

PACS numbers: PACS numbers: 75.10.Jm, 74.20.Mn 71.10.-w

\section{INTRODUCTION}

One of the central open questions in the study of frustrated systems is what happens when a classically highly degenerate magnet is subjected to violent quantum fluctuations. A model system which has played an important role in the discussion of this question is the nearest neighbour spin $1 / 2$ Heisenberg antiferromagnet on the pyrochlore lattice. This lattice consists of corner-sharing tetrahedra and exhibits a massive classical degeneracy $\underline{\underline{1}}$

Very little is known reliably about the quantum model: exact solutions are unavailable in $d=3+1$, Monte Carlo simulations are frustrated by the sign problem, and the pyrochlore lattice - being three dimensional and having a unit cell of four spins - does not yet lend itself to exact diagonalisations.

In an important, if somewhat cryptic, initial piece of analytical work back in 1992,2 Harris, Berlinsky and Bruder (HBB) took the approach of considering the bonds belonging to one of the two sublattices - "weak bonds" - of tetrahedra perturbatively (the tetrahedra of the pyrochlore lattice are arranged on the bipartite diamond lattice, which in the current context can usefully be thought of as two interpenetrating face-centred cubic lattices, see Fig. (1). The tetrahedra on the other sublattice, where the "strong bonds" reside, are thus initially decoupled, with doubly degenerate dimerised singlet ground states, parametrised by a pseudospin $\sigma$. The basic idea of HBB is then to switch on the weak bonds perturbatively, thus generating an effective interaction between the $\sigma \mathrm{s}$ on different sites. This interaction determines the eventual ground state, and in itself defines a difficult quantum problem.

A number of authors have since developed ideas based on expansions for pseudospins on an fcc lattice $\frac{3.4 .5}{{ }^{3.5}}$
Tsunetsugu's detailed work extended the scope of the mean-field theory, ${ }^{3}$ while Berg, Altman and Auerbach implemented a sophisticated numerical procedure projecting the Hamiltonian onto this Hilbert space. $\frac{5}{5}$

These approaches have in common that their starting point has a lower symmetry than the initial Hamiltonian, as a distinction between the two sublattices of tetrahedra discards the inversion symmetry of the pyrochlore lattice. In the same spirit, a perturbation theory in the weak bonds but for the full density matrix, which unlike the previous papers does not impose a restriction to the dimerised singlet subspace, at any rate found spin ordering to be absent but did not study the presence of bond ordering .6

In this paper, we take a different route by considering a Hamiltonian with an enlarged internal, rather than reduced point, symmetry: we study the spin- $1 / 2$ problem on the pyrochlore lattice via a large- $N$ quantum dimer model approach. This approach does not expressly break the symmetry between the two sublattices of tetrahedra. It provides an (artificial) small parameter, $1 / N$, which we will use to obtain an analytical solution to the first non-trivial order of the quantum dimer model.

Very unusually, we find that the quantum dimer model generated at $O(1 / N)$ is exactly soluble $\underline{\underline{z}}$ There turns out to be a phase transition in the Ising univsersality class at a temperature $T \sim O(1 / N)$, where the inversion symmetry of the pyrochlore lattice is spontaneously discarded. The set of states selected at this order preserves an extensive entropy.

The question what happens within this subspace at higher order in $1 / N$ is closely analogous to that posed in Refs. 2 34 5, as the respective starting Hilbert spaces are isomorphic. Our approach thus provides a way of reaching this starting point dynamically, i.e. by spontaneous rather than explicit symmetry breaking. 
We then show that the effective Hamiltonians obtained in Refs. 2345 belong to a family which has a simple interpretation as dimer potential terms in such an approach; as the natural basis beyond $O(1 / N)$ is not a dimer one, however, they define a quantum Hamiltonian.

These Hamiltonians have been studied using different approaches ${ }^{2,3,4.5}$ We show that a supertetrahedral ordering pattern proposed by HBB and Tsunetsugu can be represented by a simple maximally flippable dimer configuration. We also show that, within the framework of the mean-field theory, there are further configurations close by in energy, leading to numerically small characteristic energies. This implies that if there is translational symmetry breaking, the final ordering pattern might not be assumed until a temperature low compared to finite-size gaps in exact diagonalisations or to other perturbations in real compounds.

Our results thus provide an intuitive picture of the dominant physics captured in a class of theories proposed for the pyrochlore quantum antiferromagnet. As a byproduct, our results also imply that a classical dimer model on the pyrochlore lattice with a simple potential term will have an inversion and translation symmetry broken ground state with residual entropy.

In the remainder of this paper, we first introduce the $\operatorname{Sp}(N)$ quantum dimer model (Sect. III), followed by its exact solution (Sect. [II). In Sect. [IV we discuss the structure of the problem at higher orders in $1 / N$ and in Sect. V make a connection to previous work via the idea of maximally flippable dimer configurations. After some remarks on further extensions (higher-order loops, other lattices and SU(2) spins, Sects. VI VIII), we close with a short discussion of further questions raised by this approach.

\section{THE $\operatorname{Sp}(N)$ DIMER MODEL}

An $\operatorname{Sp}(N)$ dimer model is obtained from an $\mathrm{SU}(2)$ spin problem via enlarging the symmetry of spin space from $\mathrm{SU}(2) \sim \mathrm{Sp}(1)$ to $\mathrm{Sp}(N)$. Rewriting the Heisenberg Hamiltonian in terms of operators $s_{i j}^{\dagger}$, which create a singlet on the bond between spins $i$ and $j$, gives

$$
H=-J \sum_{\langle i j\rangle}\left\{s_{i j}^{\dagger} s_{i j}-\frac{1}{4 N^{2}}\right\},
$$

where $N=1$ for $\mathrm{SU}(2) \sim \mathrm{Sp}(1)$. The same form of $H$ holds for the generalisation to $\operatorname{Sp}(N)$, with $s_{i j}^{\dagger}$ now creating $\operatorname{Sp}(N)$ singlets. Details for this and the following steps are provided in the Appendix.

\section{A. The dimer model at leading order}

A hardcore dimer model is obtained from this Hamiltonian at leading order ${ }^{8}$ as one takes $N \rightarrow \infty \cdot \frac{9}{\underline{9}}$ At this or- der, any nearest-neighbour dimer covering is an orthogonal, degenerate eigenfunction of the $\operatorname{Sp}(N)$ Hamiltonian, with the ground state energy per site of $E_{0}=-J / 2$.

The ground state is thus exponentially degenerate in the volume of the system, although the precise value of the degeneracy for three dimensional lattices is not known. As the temperature is lowered below $T \sim 1$, the $\operatorname{Sp}(N)$ magnet enters the dimer manifold of ground states. It is possible in principle that this manifold already incorporates some form of order, in which case this would happen via a phase transition. However, it appears more likely that the correlations averaged over the dimer manifold remain short-ranged for the case of the nonbipartite pyrochlore lattice, 10 in which case the restriction to the dimer manifold has the form of a crossover.

The dimer wavefunctions obtained here are isotropic in spin space. This means that any further symmetry breaking occurring at subleading order can only be of a spatial variety.

\section{B. Derivation of the quantum dimer model}

The degeneracy between different dimer coverings exists only at leading order; at higher order, the model acquires a non-trivial quantum dynamics. This dynamics is determined along the lines pioneered by Rokhsar and Kivelson in the context of the SU(2) Heisenberg model ${ }^{11}$ They derived a Hamiltonian in the space of dimer coverings by formally carrying out an expansion in a parameter $x$ which in fact has the finite value of $1 / 2$, as we will briefly describe below ${ }^{11.12}$ In the $\operatorname{Sp}(N)$ model, this parameter is (artificially but) truly small: $\varepsilon \equiv 1 / 2 N$. The derivation of the dimer Hamiltonian is hence completely analogous, albeit rigorously organised, in the orders of the small parameter. Details are again given in the appendix.

At $O\left(\varepsilon^{1}\right)$, the resulting quantum dimer Hamiltonian is very simple. It reads:

$$
H_{Q D M}=2 J \varepsilon \square .
$$

Here, $\square$ stands for a resonance term around a closed loop of length four, i.e. a kinetic term which exchanges occupied and empty links if they alternate around such a loop. Note that this quantum dimer model, unlike the Rokhsar-Kivelson one, ${ }^{11}$ only contains a kinetic term. 8

\section{EXACT SOLUTION OF THE QDM: PARTIAL ORDER BY DISORDER}

The quantum dynamics induced by Eq. 2.2 can only lead to resonances between two dimer configurations which can be transformed into one another by moving exactly two dimers. Such moves are only possible for two dimers on a single tetrahedron, as the shortest closed loop not confined to a single tetrahedron has length six and therefore would require moving three dimers in order 


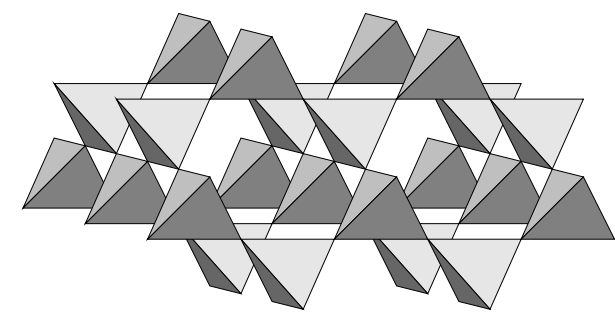

FIG. 1: The pyrochlore lattice, a network of corner-sharing tetrahedra. The tetrahedra on the two ('up' and 'down') sublattices are shaded differently. The centres of the tetrahedra of either sublattice form a face-centred cubic lattice.

to satisfy the hardcore constraint in both the initial and final configurations. On a single tetrahedron, there are three possible dimerisations in $\operatorname{Sp}(N)$, and the Hamiltonian matrix elements between them read, at this order:

$$
H_{t e t}=2 J \varepsilon\left(\begin{array}{lll}
0 & 1 & 1 \\
1 & 0 & 1 \\
1 & 1 & 0
\end{array}\right) .
$$

This matrix has one non-degenerate eigenvalue $4 J \varepsilon$ with corresponding eigenvector $\left|\phi_{A}\right\rangle=(1,1,1) / \sqrt{3}$. The other two eigenvalues are degenerate at $-2 J \varepsilon$ and eigenvectors $\left|\phi_{+}\right\rangle=\left(1, \chi, \chi^{2}\right) / \sqrt{3}$ and $\left|\phi_{-}\right\rangle=\left(1, \chi^{2}, \chi\right) / \sqrt{3}$, with $\chi \equiv \exp (2 \pi i / 3)$. These two eigenvectors form an $E$ representation of the tetrahedral group $T_{d}$. Note that the asymmetry of the spectrum under $J \leftrightarrow-J$ is a manifestation of our inability - in contrast to the case of the square $\frac{11}{11}$ and triangular ${ }^{13}$ lattices - to choose the sign of the overlap matrix elements at will.

A single tetrahedron at this order is thus occupied by 0 or 1 dimers and gains no energy from resonance moves, or it is occupied by 2 dimers, in which case it gains an energy $-2 J \varepsilon<0$. We thus need to maximise the number of tetrahedra occupied by two dimers.

As the number of dimers equals the number of tetrahedra, this is done by putting no dimers on one half the tetrahedra, and two each on the remaining tetrahedra. As two tetrahedra with two dimers each cannot be neighbours, and as the tetrahedra reside on the bipartite diamond lattice, this implies that one sublattice of tetrahedra, containing the 'down' tetrahedra, say, is empty, whereas the 'up' tetrahedra have two dimers each. This gains an energy of $-2 J \varepsilon$ per up tetrahedron, and hence $-J \varepsilon / 2$ per site.

The quantum dimer model up to this order thus spontaneously breaks the inversion symmetry through the sites of the pyrochlore lattice (this operation exchanges up and down tetrahedra); the point group is broken down from $O_{h}$ to $T_{d}$. This is, to our knowledge, the first model where such a symmetry breaking can be demonstrated to occur; this feature is not unimportant as most treatments use this symmetry breaking as part of the starting assumptions. We also note that this is a rare case where a quantum dimer model is analytically soluble - normally, finding the solution is a hard problem requiring intensive numerics in itself $?$
Despite this symmetry breaking, the ground state at $O\left(\varepsilon^{1}\right)$ retains a large degeneracy as each single tetrahedron continues to exhibit a two-fold degeneracy; the residual entropy per spin is hence $\mathcal{S}=\left(k_{B} / 4\right) \ln 2$, where $k_{B}$ is the Boltzmann constant.

We thus find a transition at which the point group is reduced by discarding inversion symmetry, but spin rotational as well as real space translational symmetries remain intact. This is an example of (only partial) order by disorder in a quantum frustrated system.

\section{THE FATE OF THE RESIDUAL DEGENERACY}

So far, to $O(\varepsilon)$, we have been fortunate in dealing with a quantum dimer model with a simple structure. Configurations differing in the distribution of dimers between tetrahedra define disconnected dynamical sectors, the ground-state energies of which are given by the number of doubly-occupied tetrahedra, with the corresponding wavefunctions being outer products over tetrahedron wavefunctions. Note that such a simple structure is generically absent for other lattices, the leading dimer model typically not being exactly soluble.

Here, these problems are deferred to the next order, $\varepsilon^{2}$, where the degrees of freedom are given by one pseudospins- $1 / 2$ for each doubly occupied tetrahedron. For the ground-state sector, these pseudospins reside on the face-centred cubic lattice defined by one sublattice of tetrahedra. These pseudospins encode the $E$ representation of $T_{d}$ provided by the degenerate wavefunctions $\left|\phi_{ \pm}\right\rangle$.

Nonetheless, further insight can be gained by continuing to use a dimer basis, on the understanding that a diagonal operator in the dimer basis will not be diagonal in the pseudospin basis. This approach will lead us to a simple interpretation of the effective Hamiltonians of Refs. 23 4,5. Such a connection is possible as, formally, the pseudospins on a face-centred cubic lattice are also the starting point of those studies, where the three (linearly dependent) SU(2) dimer coverings of an isolated tetrahedron reduce to a two-dimensional $E$ representation.

\section{A. Effective Hamiltonians}

Let us consider the possible dimer operators we can write down beyond the ones for the loops of length 4 already included at $O(\varepsilon)$. The next simple kinetic term is that for a hexagon, $\square$, which is in principle generated at $O\left(\varepsilon^{2}\right)$. However, such a term moves dimers from the occupied sublattice of tetrahedra to the unoccupied one, and thus does not have a matrix element of $O(1)$ within the pseudospin subspace in which we are doing degenerate perturbation theory. It will, however, contribute via a 'virtual' process by first shifting three dimers onto 
the empty tetrahedra and then back again. This process is diagonal in dimer basis, pictorially represented by a potential term of the form

$$
H_{\square}=-\sum_{\square}\left|{ }_{-}^{\prime}\right\rangle\left\langle{ }_{-}^{\prime}\right\rangle,
$$

where the symbol $\left|{ }_{-}^{\prime}\right\rangle\left\langle\left\langle{ }_{-}\right\rangle\right.$stands for an operator which projects out the states with zero amplitude of dimer occupancy on the three bonds of the occupied tetrahedra of a given hexagonal loop. Here, the symbol $\sum_{\square}$ implies a sum over all such hexagonal loops.

The physics of this term is most transparent in the dimer basis. However, it is off-diagonal in the basis of states we are doing perturbation theory in. Let us therefore rewrite the dimer potential term in pseudospin basis.

To do this, we write the wavefunction on each occupied tetrahedron in terms of two angles, $\alpha$ and $\theta$ :

$$
\begin{aligned}
|\theta, \alpha\rangle \equiv & \sin (\alpha / 2) \exp (i \theta / 2)\left|\phi_{+}\right\rangle \\
& +\cos (\alpha / 2) \exp (-i \theta / 2)\left|\phi_{-}\right\rangle .
\end{aligned}
$$

Next, we observe that the probability of finding a dimer on a given link of the tetrahedron is given by

$$
\left[1+\sin \alpha_{i} \cos \left(\theta_{i}-\nu_{\mathrm{o}, i}\right)\right] / 3,
$$

where $\nu_{\mathrm{o}, i}=0, \pm 2 \pi / 3$ according to which of the three possible dimer pairings is selected.

Using this parametrisation, the potential term for the pseudospins on the face-centred cubic lattice has the form

$$
H_{f c c}=-\sum_{\square} \prod_{i=1}^{3}\left[1+\sin \alpha_{i} \cos \left(\theta_{i}-\nu_{\mathrm{o}, i}\right)\right] / 3 .
$$

Here, the angles $\nu_{0, i}$ depend on the loop under consideration and are such that $\theta=\nu_{\mathrm{o}, i}$ together with $\alpha=\pi / 2$ corresponds to the state which maximises the dimer amplitude on the link which forms part of the hexagonal loop. Put another way, the factors in the product are the probabilities of finding dimers on the respective links. The sum on $i$ runs over the tetrahedra to which these links belong.

This form can in turn be rewritten by introducing pseudospins (represented by Pauli matrices $\sigma$ ) on each tetrahedron with the quantisation axis along the azimuthal axis $\alpha=0$, and $\hat{e}_{\mathrm{o}, i}$ denoting the preferred directions $\theta=\nu_{\mathrm{o}, i}$ and $\alpha=\pi / 2$ :

$$
H_{f c c}=-\sum_{\square} \prod_{i=1}^{3}\left[(1+\Upsilon)+\vec{\sigma} \cdot \hat{e}_{\mathrm{o}, i}\right] / 3 .
$$

For the hexagonal potential, $H_{\square}$, one has $\Upsilon=0$. $\Upsilon \neq$ 0 corresponds to an additional potential term involving only two dimers on a hexagon, pictorially represented as

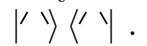

The form of $H_{f c c}$ is in fact the same as that of the effective Hamiltonians obtained in Refs. 2 3. 4 .5. The intermediate steps in the algebra differ between $\operatorname{Sp}(N)$ and
$\mathrm{SU}(2)$ on account of the non-orthogonality of the dimerisations of a tetrahedron in SU(2) [see Sect. VII]. Most importantly, the $\nu_{\mathrm{o}, i}$ are shifted by $\pi$.

However, in the end the effective SU(2) Hamiltonians correspond to dimer potentials like $H_{\square}$ (Eq. 4.1) in the same way that $H_{f c c}$ does. This common equivalence states in a crisp geometrical form that both the $\mathrm{Sp}(N)$ (Eq. 4.5) and the $\mathrm{SU}(2)$ effective Hamiltonians of Refs. 2, 4,5 encode dimer potentials.

\section{B. Mean-field theory for $H_{f c c}$}

Even with the restriction to this class of Hamiltonians, one is still left with the hard problem of minimising $H_{f c c}$, which has the form of a $S=1 / 2$ Heisenberg model with spin-orbit coupling.

A proper quantum mechanical treatment of this problem is beyond the scope of this paper. Rather, to shed some light on the physics unearthed in previous studies, we considered this problem in a mean-field theory, treating the pseudospins- $1 / 2$ as being effectively classical ${ }^{2,3,4}$

For $\Upsilon=0$, the best state we have discovered so far has a four sublattice structure, as do the states of Refs. 2.3, 4.5 , with angles $\alpha \equiv \pi / 2$ and $\left\{\theta_{i}\right\}=\{0,0, \pi-\delta, \pi+\delta\}$, where $\delta=\arccos (7 / 8) \approx 0.5054$.

This state has an energy per loop (and therefore per spin), $\lambda$, with $\lambda=-289 / 3456 \approx-0.08362$. The highest possible energy, realised for $\theta_{i} \equiv \pi$, gives $\lambda=0$. Even a state with $\delta$ significantly different can have a closeby energy: for example, for $\delta=0$, one obtains $\lambda=-1 / 12 \approx$ -0.08333 . This state has a higher symmetry as it only represents a two-sublattice ordering.

As $\Upsilon$ is changed, $\delta$ evolves, and it reaches $\delta=\pi / 3$ at $\Upsilon=-1 / 2$; at this point, it in fact becomes possible to disorder one of the four sublattices at zero cost in energy; this we call Harris-Bruder-Berlinsky-Tsunetsugu (HBB-T) state. For $\Upsilon=0$, this configuration $\left\{\theta_{i}\right\}=$ $\left\{0, \theta_{2}, 2 \pi / 3,-2 \pi / 3\right\}$ is no longer optimal in our case but still rather close: $\lambda=-35 / 432 \approx-0.08102$.

These results raise two points. The first is the observation that the particular four-sublattice ordering with one disordered sublattice is not entirely generic, as even in mean-field theory, it does not correspond to an extended parameter region in $\Upsilon$.

The second is the recurring theme of the emergence of a numerically small energy scale. There are configurations the symmetry properties of which differ significantly but whose energies are very similar. The configurations discussed above are degenerate at $O\left(\varepsilon^{0}\right)$ and $O\left(\varepsilon^{1}\right)$, and their variational energies with respect to $H_{f c c}$ differ only by a few percent.

These facts suggest that the magnet will break translational symmetry - by assuming whatever ordered configuration it chooses - only at very low temperatures, provided quantum fluctuations do not prevent this ordering altogether. 


\section{THE HBB-T STATE AS A DIMER STATE}

Given the Hamiltonian $H_{f c c}$ is most simply written in dimer basis, it is natural to ask whether the mean-field ground states have a natural interpretation in terms of dimer coverings. This is most pertinent for states with angles $\alpha=\pi / 2$ and $\theta$ integer multiples $2 \pi / 3$, as these correspond to maximal dimer amplitudes on one of the three pairs of links,,$\frac{3}{3}$ and are thus naturally identified with a dimer configuration.

The HBB-T state in fact has a very simple such interpretation: it can be represented as the dimer configuration with the maximal number of flippable hexagons, that is to say as the ground state of the classical dimer model with a hexagonal potential of the type $H_{\square}$ [Eq. [4.1)]. Why one sublattice of tetrahedra remains disordered for the maximally flippable configuration also becomes apparent in this language.

As an aside, we note that the appearance of such maximally flippable configurations is a generic feature of quantum frustrated systems. In a nutshell, a large number of flippable loops implies a wide range of possible fluctuations, and hence undetermined degrees of freedom. These the perturbation can make use of by arranging them to its liking. Such a route to quantum order by disorder ${ }^{14}$ has been discussed in detail in the context of frustrated transverse field Ising models $\underline{15}$

To demonstrate this, we first need to show which classical dimer states optimise this problem; then, we render the HBB-T state in a way which makes its identity manifest.

\section{A. Maximally flippable configurations}

To maximise the number of flippable hexagons, $n_{\square}^{f l}$, we note that the hexagons lie in the [111] kagome planes, and that each bond of the pyrochlore lattice is part of hexagons in two different planes, e.g. [111] and [-1-11]. Thus, each dimer can be part of at most two flippable hexagons; as the number of hexagons, $n_{\square}$, equals the number of spins, and hence twice the number of dimers, and as three dimers are needed for a flippable hexagon, this provides an upper limit of $n_{\square}^{f l} / n_{\square}=1 / 3$.

However, it turns out to be impossible to saturate this bound, as can be seen by explicitly constructing configurations which are locally maximally flippable. Consider first a configuration with independently flippable loops. In this case, one needs at least three dimers per resonant loop, and one obtains for the upper bound $n_{\square}^{f l} / n_{\square}=1 / 6$. A more favourable state would be one in which two flippable hexagons share one dimer in two different kagome planes, e.g. [111] and [-111]. For such a local configuration one needs at least five dimers for each pair of resonant loops, and this yields the upper bound $n_{\square}^{f l} / n_{\square}=1 / 5$.

This local configuration might in principle be used as

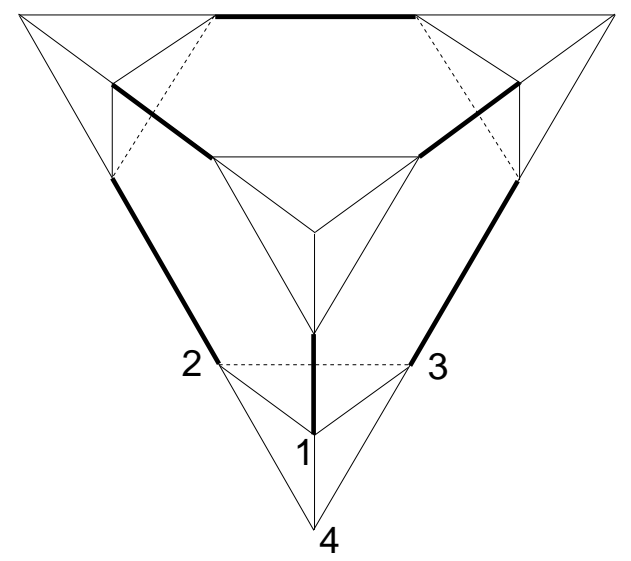

FIG. 2: A flippable supertetrahedron consists of six dimers, which form four flippable hexagons in the four $\{111\}$ kagome planes, and four surrounding dimerfree tetrahedra of the down-sublattice, such as (1234).

a building block for a new configuration if other dimers are added locally to form other flippable hexagons. There is only one possibility of adding a sixth dimer to build a third flippable hexagon, and this automatically yields a forth one, with one flippable hexagon in each kagome plane. This local configuration of four flippable hexagons, constructed by six dimers, has the form of a flippable supertetrahedron shown in Fig. 22

This flippable supertetrahedron has four dimerfree tetrahedra at its corners. None of the dimers emanating from the outer vertices of these tetrahedra can be part of a flippable hexagon, and thus at least 2 dimers are lost (four dimers each shared between two flippable supertetrahedra).

To obtain four flippable hexagons, one thus needs to invest $6+2=8$ dimers, and hence 16 sites. As the number of sites equals the number of hexagonal loops, this establishes the upper bound of

$$
n_{\square}^{f l} / n_{\square} \leq 1 / 4 .
$$

A consequence of the local flippable supertetrahedron structure is that an optimal configuration, with $n_{\square}^{f l} / n_{\square}=1 / 4$ automatically breaks the inversion symmetry, i.e. all up-tetrahedra contain two dimers and all down-tetrahedra are dimerfree.

As an aside, we note that a classical attractive potential for dimer pairs on a tetrahedron would have selected the dimer manifold (with entropy $k_{B} \ln 3 / 4$ per site) with all dimers on one sublattice of tetrahedra. The attractive hexagonal loop term (by itself or in combination with this term) in addition imposes the creation of the supertetrahedra.

\section{B. Connection to the HBB-T state}

An optimal flippable supertetrahedron tiling is shown in Fig. 3. The dimers which do not take part in the 

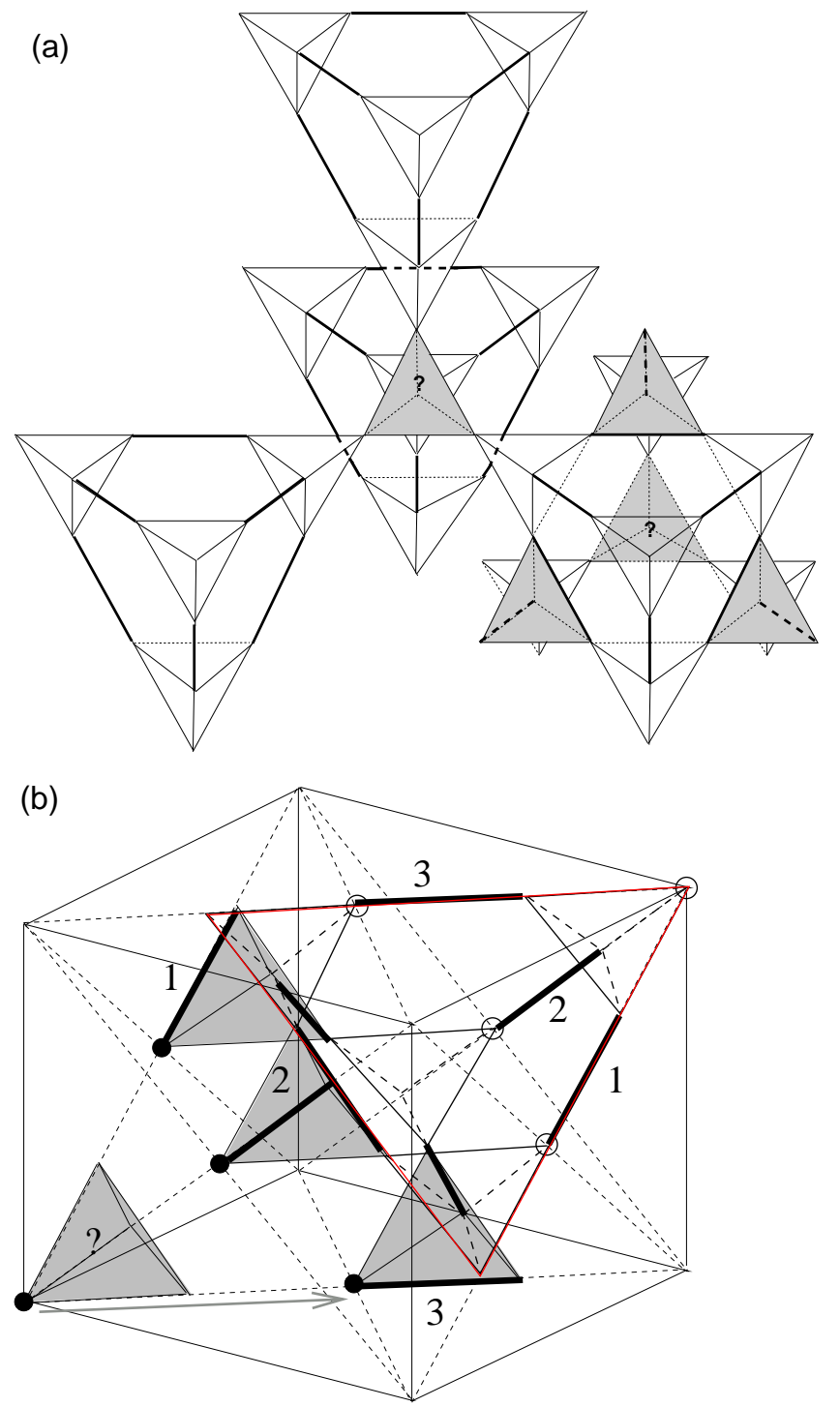

FIG. 3: Optimal dimer configuration. (a) Gray tetrahedra form the up-sublattice, and white tetrahedra (dimerfree) form the down-sublattice. Four flippable supertetrahedra meet in one ?-tetrahedron of the up-sublattice. The dimer configurations of these ?-tetrahedra may be chosen arbitrarily. The flippable supertetrahedron on the right hand side is represented in such a manner to make clear the relation to the mean-field configuration of Ref. 3. (b) Cubic lattice cell of the flippable supertetrahedron configuration. The flippable supertetrahedron is shown in red.

flippable supertetrahedron formation are found on the ?-tetrahedra in Fig. 3a and may be chosen freely. The ground-state degeneracy of the optimal configuration therefore remains macroscopic, and the entropy per site is $\mathcal{S}_{\varepsilon^{2}}=\left(k_{B} / 16\right) \ln 2{ }^{3}$ Because the up-tetrahedra form an fcc-lattice, the ?-tetrahedra now live on a cubic lattice and connect the flippable supertetrahedra. This is reminiscent of the HBB-T state; indeed, the cartoon for this state is precisely our state of flippable supertetrahedra, see Fig. 3. where we have shown the flippable superte-

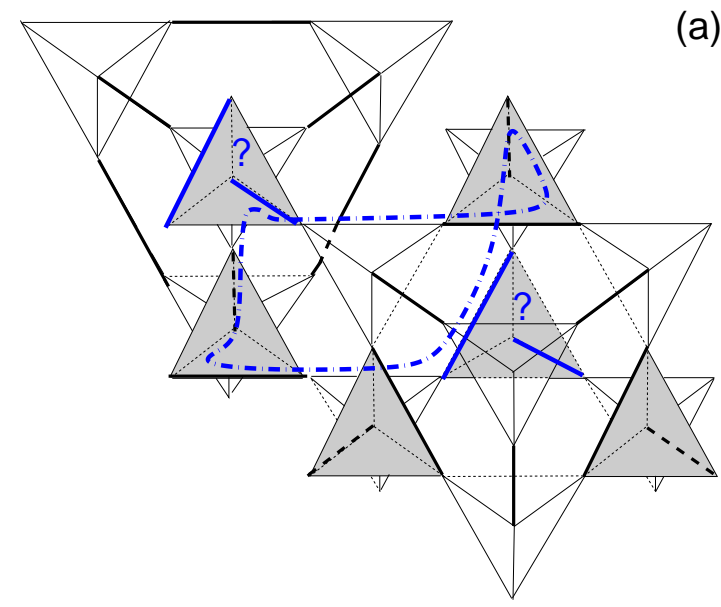

FIG. 4: Twelve-bond loop connecting two nearest neighbours of ?-tetrahedra (blue dashed-dotted line). The dimerisation of the connected ?-tetrahedra has to be the same (here: blue).

trahedra, together with the dimer configurations on the shaded tetrahedra as dictated by the HBB-T state. However, by focussing on the up-tetrahedra, rather than the flippable loops, the geometry is harder to visualise.

One may ask whether there exist other tilings of flippable supertetrahedrawhich are also optimal. Indeed, if one chooses the ?-tetrahedron in the cubic lattice cell in Fig. 3b in such a manner that its dimerisation coincides with that of the tetrahedron at lattice point [110] (3dimerisation), one may displace one layer of cubes with respect to a neighbouring layer in the [110] direction, as indicated by the gray arrow.

However, this one choice forces us to fix the dimerisation of all ?-tetrahedra at the interface of the displacement. The resulting interface energy will thus generically win over the displacement entropy and lift this degeneracy.

\section{HIGHER-ORDER LOOPS}

In the absence of an exact solution at $O\left(\varepsilon^{2}\right)$, our present approach does not per se justify pursuing the physics of longer loops. For completeness, however, we mention some geometric facts about the entropics of the residual degrees of freedom in the dimer model with the uniform four-sublattice ordered state, with one disordered sublattice, as a starting point. We will discuss this topic more completely separately 16

The as yet undetermined dimers can only participate in virtual resonance loops of length 12 (involving moving six dimers). One, rather contorted loop, can connect a pair of nearest-neighbour ?-tetrahedra (Fig. 4). The other involves three ?-tetrahedra which are mutually next-nearest neighbours. This is displayed in Fig. 5 .

The nearest-neighbour loop can only (virtually) resonate if the dimers are oriented perpendicular to the 


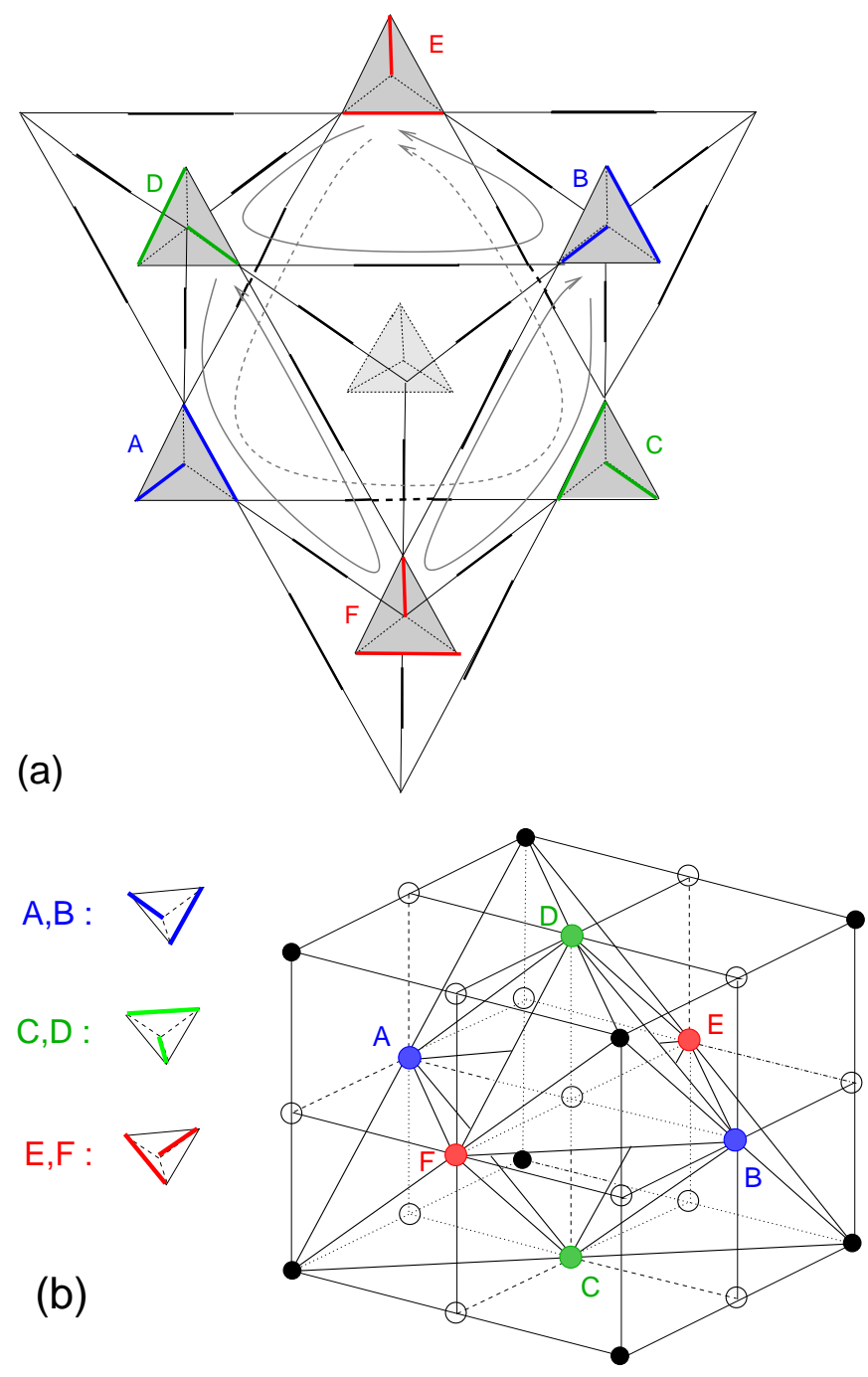

FIG. 5: Next-nearest-neighbour loops. (a) Closest packing of 12-bond loops connecting next-nearest neighbours of ?tetrahedra (dark gray). The tetrahedron in light gray is part of the other square sublattice. The superstructure is again an flippable supertetrahedron, but with unequal bonds (flippable (super) ${ }^{2}$ tetrahedron). (b) Elementary lattice cell of the flippable (super) ${ }^{2}$ tetrahedron configuration. The ?tetrahedra on the cubic sublattice, which are connected by next-nearest-neighbour loops, are represented by full circles (black and coloured). The open circles represent ?-tetrahedra on the second cubic sublattice. The four resonant nextnearest-neighbour loops are those connecting $A F D, B E D$, $B F C$, and $A E C$.

line joining the ?-tetrahedra, and it thus favours the formation of rods of ?-tetrahedra with identical alignment, somewhat reminiscent of the supertetrahedral ordering pattern found in Ref. 5 .

The next-nearest neighbour loops only connect tetrahedra living on the same sublattice of the (bipartite) cubic lattice on which the ?-tetrahedra reside. Trying to maximise the number of flippable next-nearest neighbour loops thus leads to two identical copies of the same problem. Amusingly, the maximally flippable n.n.n.loop configuration is self-similar: one now finds flippable (super) $)^{2}$ tetrahedra, with one out of four ?-tetrahedra remaining again undetermined (Fig. 50).

The maximal total number of flippable loops of length 12 is obtained by taking the two copies of this flippable $(\text { super })^{2}$ tetrahedron state and arranging the ?-tetrahedra to satisfy the rod-arrangement dictated by the nearest neighbour loops.

\section{THE SU(2) LIMIT}

The Rokhsar-Kivelson quantum dimer model for SU(2) spins is based on an expansion in the same small parameter, $\varepsilon$, appearing in the overlap matrix. However, this parameter is not in fact infinitesimal $-1 / 2$, in fact - and this leads to a certain arbitrariness in assigning an order to a given matrix element as a change in its order can be offset by a numerical factor 2 . This is most plainly seen for the case of the constant $1 / 4 N^{2} \sim O\left(\varepsilon^{2}\right)$ in the Hamiltonian, which in the case of $\mathrm{SU}(2)$ simply becomes a constant $1 / 4 \sim O\left(\varepsilon^{0}\right)$. Here, we evaluate the matrix elements between two dimer configurations exactly and assign them the order of the overlap between the two configurations.

On the pyrochlore lattice, the main obstacle lies in the fact that $\mathrm{SU}(2)$ dimerisations of a tetrahedron are not linearly independent. In the quantum dimer model, this shows up in the form of a non-invertible overlap matrix $S$. In such a situation, Eq. A5 is no longer meaningful.

One can adopt two different stances with respect to this observation. Firstly, one can hope that - by continuity - the large- $N$ physics will nonetheless be relevant to the $\mathrm{SU}(2) \sim \mathrm{Sp}(N=1)$ case. Alternatively, one can decide that $\mathrm{SU}(2)$ is special, and that the physics discussed so far in this paper will qualitatively have to be modified for the $N=1$ case.

Here, it is useful to note that one also finds that the resonance energy of a single tetrahedron vanishes - for the $\mathrm{SU}(2) \mathrm{RK}$ model, the matrix elements in Eq. 3.1 are zero. Let us thus sidestep the orthogonality issue by ignoring the length four loops altogether, with a leading term of the QDM proportional to the hexagonal resonance term $-J \varepsilon^{2} \bigcirc$. This is fundamentally different from $H_{f c c}$ in that it represents a kinetic, and not a potential, dimer term. The ground state of this quantum dimer model (and whether it breaks the lattice inversion, or indeed any other, symmetry) is not known. Candidate variational states for this problem can be constructed along the lines explored in Ref. 15. One such candidate is the maximally flippable supertetrahedron-configuration discussed above.

As the flippable hexagons there are not independently flippable, other candidate states can be constructed to maximise the number of maximally flippable hexagons; one example can be obtained from the proposal of Ref. 17 . This has one in six hexagons flippable and does not break 
the inversion symmetry. We leave a detailed analysis of this quantum dimer model as a subject for future study.

\section{OTHER LATTICES}

The approach developed here can easily be extended to other lattices. However, the exact solubility discovered here is in general not encountered elsewhere. In fact, the situation can differ from the pyrochlore one in several respects $\underline{16}$

Firstly, the lattice under consideration may not admit any dimer coverings. Secondly, all dimer states may have a fixed number of flippable shortest loops of even length, thereby rendering the leading order dimer model trivial. Thirdly, the shortest loops may be inequivalent or overlapping in a way so as to destroy the solubility. In addition, the appropriate low-energy sector may at any rate be better described by a different type of effective model 18

\section{CONCLUSION}

In conclusion, we have presented an exactly soluble deformation of the highly frustrated quantum pyrochlore antiferromagnet. To our knowledge, this provides the first instance in which the inversion symmetry of the pyrochlore lattice by itself is spontaneously broken.

From an experimental perspective, this is perhaps the most interesting observation in this paper: upon lowering the temperature from the paramagnetic phase, the current scenario implies the presence of an initial Ising transition into a non-magnetic phase with finite entropy and full translational and rotational symmetries.

Of course, the presence of such a symmetry broken phase is in a sense already implicit in the starting points of Refs. 23 4 5s With respect to these, our approach has provided a simple physical picture by shedding some light on the 'natural' degrees of freedom arising in such treatments, the hexagonal resonating loops. This reinforces the idea of an non-magnetic ordering pattern with an enlarged unit cell.

Our work therefore goes some way towards providing a rationale for the sequences of symmetry-breakings discussed before ${ }^{2.3 .4 .5}$ Whether or not the scenario discussed here provides the appropriate framework for understanding the nearest neighbour $\mathrm{S}=1 / 2$ pyrochlore Heisenberg antiferromagnet remains an open question.

\section{Acknowledgements}

We would like to thank Ehud Altman, Assa Auerbach, Benoit Douçot, Subir Sachdev and Hirokazu Tsunetsugu for useful conversations. We are very grateful to Oleg Tchernyshyov for those and for collaboration on closely related work, and to Steve Kivelson for sharing his unpublished notes. RM is grateful to the Aspen Center for Physics and the Lorentz Center of Leiden University, where part of this work was carried out. He is supported in part by by the Ministère de la Recherche et des Nouvelles Technologies with an ACI grant. MOG is supported by the Swiss National Foundation for Scientific Research under grant No. PBFR-106672. SLS would like to acknowledge support by the NSF (DMR-9978074 and 0213706) and the David and Lucile Packard Foundation.

\section{APPENDIX A: THE $\operatorname{Sp}(N)$ DIMER MODEL}

As a first step, one introduces bosonic operators ("Schwinger bosons") $\left\{b_{\uparrow}, b_{\downarrow}\right\}$, to represent the spin operators in the following way: $S^{z}=\left(b_{\uparrow}^{\dagger} b_{\uparrow}-b_{\downarrow}^{\dagger} b_{\downarrow}\right) / 2$, $S^{+}=b_{\uparrow}^{\dagger} b_{\downarrow}$. In order to represent $\mathrm{SU}(2)$ spins $1 / 2$, one needs to supplement the bosonic description with the constraint $n_{b} \equiv b_{\uparrow}^{\dagger} b_{\uparrow}+b_{\downarrow}^{\dagger} b_{\downarrow}=1$ on each site.

In terms of these operators, the antiferromagnetic nearest-neighbour Heisenberg Hamiltonian becomes $(J>$ $0)$ :

$$
\begin{aligned}
H & =J \sum_{\langle i j\rangle} \mathbf{S}_{i} \cdot \mathbf{S}_{j} \\
& =-J \sum_{\langle i j\rangle}\left\{\left(\epsilon^{\sigma \tau} b_{i \sigma}^{\dagger} b_{j \tau}^{\dagger} / \sqrt{2}\right)\left(\epsilon^{\mu \nu} b_{i \mu} b_{j \nu} / \sqrt{2}\right)-1 / 4\right\},
\end{aligned}
$$

where $\mathbf{S}_{i}$ are spin 1/2 SU(2) operators. This expression is formally generalised to $\operatorname{Sp}(N)$ by the introduction of an additional flavour index for the bosons, which we label by a capital letter. $\operatorname{In} \operatorname{Sp}(N)$, there are $N$ such flavours; the case of $\mathrm{SU}(2)$ corresponds to one flavour: $\mathrm{SU}(2) \sim \mathrm{Sp}(1)$. The $\operatorname{Sp}(N)$ generalisation, $\mathcal{J}_{A B}^{\mu \nu}$, of the Levi-Civita symbol continues to be off-diagonal and antisymmetric in the spin index but simply diagonal in the flavour index: $\mathcal{J}_{A B}^{\mu \nu}=\epsilon^{\mu \nu} \delta_{A B}$. We define the singlet (hereafter called dimer) annihilation operator for bond $\langle i j\rangle$ :

$$
s_{i j} \equiv \mathcal{J}_{A B}^{\sigma \tau} b_{i \sigma}^{A} b_{j \tau}^{B} / \sqrt{2 N},
$$

where summation over spin and colour indices is implicit. The $\operatorname{Sp}(N)$ Hamiltonian reads

$$
H=-J \sum_{\langle i j\rangle}\left\{s_{i j}^{\dagger} s_{i j}-\frac{1}{4 N^{2}}\right\} .
$$

\section{The dimer model at leading order}

A hardcore dimer model is obtained from this Hamil-

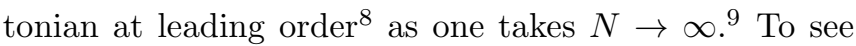
this, consider the properties of dimer coverings of the lattice. Let $P$ denote an ordered pairing of the sites of the lattice and $\left|\psi_{P}\right\rangle$ the corresponding singlet wavefunction: $\left|\psi_{P}\right\rangle \equiv \prod s_{p_{2 n-1} p_{2 n}}^{\dagger}|0\rangle$, where $|0\rangle$ is the state with no 
bosons present and $p_{2 n-1}$ and $p_{2 n}$ are the two members of the $n^{\text {th }}$ pair contained in $P$. $n$ runs from 1 to $N_{s} / 2$, where $N_{s}$ denotes the number of sites.

Any such state $\left|\psi_{P}\right\rangle$ satisfies the constraint $n_{b}=1$ for every site. At leading order, $O\left(1 / N^{0}\right)$, these coverings are orthogonal, degenerate eigenfunctions of the $\operatorname{Sp}(N)$ Hamiltonian: $H\left|\psi_{P}\right\rangle=E_{0}\left|\psi_{P}\right\rangle$, with the ground state energy $E_{0}=-J N_{s} / 2$. Due to the constraint on the boson number, it is not possible to have more than one dimer per site, and due to the form of the Hamiltonian, it is disadvantageous to have less. At $O\left(1 / N^{0}\right)$, the ground states are thus all the hardcore dimer coverings, denoted by $P$, of the lattice.

\section{Derivation of the quantum dimer model}

As the different dimer coverings are not exactly orthogonal, one obtains an overlap matrix, $S$, between different coverings which has the following schematic form:

$$
S=\mathbb{1}-\varepsilon \square+\varepsilon^{2} \square+\varepsilon^{2} \square \times \square+O\left(\varepsilon^{3}\right) .
$$

Here, $\mathbb{1}$ is the unit matrix, and the symbols $\square, \square$ denote a nonvanishing matrix element between two dimer configurations differing in the positions of two and three dimers, respectively.

The signs of the overlap matrix elements cannot be chosen freely; in fact, there is no convention which uniformly yields a positive sign in front of the $\square$ term. This is in contrast to the case of the square and triangular lattices, where this choice is a matter of convention. ${ }^{11.13}$
However, one is free to choose the sign of the $\square$ terms to be, for example, all positive or all negative.

We can now formally orthonormalise the basis set by introducing basis states $\left|\Psi_{p}\right\rangle \equiv \sum_{p^{\prime}}\left(S^{-1 / 2}\right)_{p p^{\prime}}\left|\psi_{p^{\prime}}\right\rangle$. Here, $S^{-1 / 2}$ is the (symmetric) inverse square root of $S$, which can be obtained from $S$ by a Taylor expansion in $\varepsilon$. The $\left|\Psi_{p}\right\rangle$ can be labelled uniquely by a parent dimer configuration $P$, as the other dimer states are admixed only to higher order in $\varepsilon$. Explicitly, orthonormality follows from

$$
\begin{aligned}
\left\langle\Psi_{p} \mid \Psi_{q}\right\rangle & =\sum_{p^{\prime} q^{\prime}} S_{p p^{\prime}}^{-1 / 2} S_{q q^{\prime}}^{-1 / 2}\left\langle\psi_{p^{\prime}} \mid \psi_{q^{\prime}}\right\rangle \\
& =\left(S^{-1 / 2} S S^{-1 / 2}\right)_{p q}=\mathbb{1}_{p q} .
\end{aligned}
$$

The matrix elements of $H$ in the orthogonalised basis now read

$$
\begin{aligned}
\mathcal{H}_{p q} & \equiv\left\langle\Psi_{p}|H| \Psi_{q}\right\rangle=\sum_{p^{\prime} q^{\prime}} S_{p p^{\prime}}^{-1 / 2} S_{q q^{\prime}}^{-1 / 2}\left\langle\psi_{p^{\prime}}|H| \psi_{q^{\prime}}\right\rangle \\
& =\left(S^{-1 / 2} H S^{-1 / 2}\right)_{p q} .
\end{aligned}
$$

This expression is useful as it is also possible to expand $\left\langle\psi_{p^{\prime}}|H| \psi_{q^{\prime}}\right\rangle$ in powers of $\varepsilon$. It turns out to be convenient to subtract off the energy of a dimerised state, $-J\left(1-1 / 4 N^{2}\right) N_{s} / 2$, so that diagonal terms are absent: $\left\langle\psi_{p^{\prime}}|H| \psi_{p^{\prime}}\right\rangle=O\left(\varepsilon^{2}\right)$, and the expansion of the Hamiltonian matrix contains no terms of $O\left(\varepsilon^{0}\right)$. Thence,

$$
H_{Q D M}=S^{-1 / 2} H S^{-1 / 2}=2 J \varepsilon \square+O\left(\varepsilon^{2}\right) .
$$

1 P. W. Anderson, Phys. Rev.102, 1008 (1956); J. Villain, Z. Phys. B 33, 31 (1979); R. Moessner and J. T. Chalker, Phys. Rev. Lett. 80, 2929 (1998).

2 A. B. Harris, A. J. Berlinsky and C. Bruder, J. Appl. Phys. 69, 5200 (1991).

3 H. Tsunetsugu, J. Phys. Soc. Jpn. 70, 640 (2001); Phys. Rev. B 65, 024415 (2002).

4 M. Elhajal, B. Canals and C. Lacroix, Can. J. Phys. 79, 1353 (2001).

5 E. Berg, E. Altman and A. Auerbach, Phys. Rev. Lett. 90, 147204 (2004).

6 B. Canals and C. Lacroix, Phys. Rev. Lett. 80, 2933 (1998).

7 Two other types of non-trivial soluble dimer models are: dimer models at a Rokhsar-Kivelson point, ${ }^{11}$ where each kinetic term is counterbalanced by a fine-tuned potential term; and dimer models with balanced inequivalent kinetic terms [G. Misguich, D. Serban and V. Pasquier, Phys. Rev. Lett. 89, 137202 (2002)].

8 S. Sachdev, cond-mat/9303014 (Lectures presented at the summer course on Low Dimensional Quantum Field Theories for Condensed Matter Physicists 24 Aug. to 4 Sep. 1992, Trieste, Italy).
${ }^{9}$ Here, we keep $n_{b}=1$. The case of fixed $\kappa \equiv n_{b} / N$ as $N \rightarrow \infty$ is probably more familiar from the literature on frustrated magnets; see e.g. S. Sachdev, Phys. Rev. B 45, 12377 (1992).

10 D. Huse, W. Krauth, R. Moessner and S. L. Sondhi, Phys. Rev. Lett. 91, 167004 (2003).

11 D. S. Rokhsar and S. A. Kivelson, Phys. Rev. Lett. 61, 2376 (1988).

12 S. A. Kivelson, unpublished notes.

13 R. Moessner and S. L. Sondhi, Phys. Rev. Lett. 86, 1881 (2001).

14 E. F. Shender, Sov. Phys. JETP 56, 178 (1982).

15 R. Moessner, S. L. Sondhi and P. Chandra, Phys. Rev. Lett. 84, 4457 (2000); R. Moessner and S. L. Sondhi, Phys. Rev. B 63, 224401 (2001).

16 M. O. Goerbig et al., work in progress.

17 S. H. Lee, C. Broholm, W. Ratcliff, G. Gasparovic, Q. Huang, T. H. Kim and S. W. Cheong, Nature 418, 856 (2002).

18 M. Mambrini, J. Trebosc and F. Mila, Phys. Rev. B 59, 13806 (1999) . 